\title{
ANÁLISE SOBRE RISCO E VULNERABILIDADE À COVID-19 NO ESTADO DE MATO GROSSO DO SUL
}

\section{ANALYSIS OF RISK AND VULNERABILITY TO COVID-19 IN THE STATE OF MATO GROSSO DO SUL}

\author{
Mauro Henrique Soares da Silva \\ Doutor em Geografia. UFMS \\ mauro.soares@ufms.br \\ Marine Dubos Raoul \\ Doutora em Geografia. UFMS \\ marine.raoul@gmail.com \\ Diego Ribeiro Oquendo Cabrero \\ Mestrando em Geografia. UFMS \\ diego.cabrero@gmail.com
}

\begin{abstract}
RESUMO
Diante da atual situação de Pandemia declarada pela Organização Mundial da Saúde, em março de 2020, decorrente da dispersão rápida do Coronavírus, cuja doença principal - a COVID-19 - pode causar superlotação dos serviços públicos de saúde e acentuar as perdas de vida da população, surgem questionamentos relativos ao quão vulnerável os territórios estão em relação a esse fenômeno de perigosidade, e, para além disso, qual o grau de risco que as populações estão expostas, considerando as características dos territórios. Nesse sentido, o objetivo desta pesquisa foi analisar a vulnerabilidade e o risco à COVID-19 no estado de Mato Grosso do Sul, considerando as características do território, uma vez que o referido estado apresentou um aumento de $68 \%$ dos casos confirmados no período epidemiológico entre 23 e 30 de abril, atingindo 255 contaminados. Para atingir esse objetivo foram elencadas algumas variáveis específicas, com a finalidade de quantificar e analisar tanto a vulnerabilidade, quanto o risco dos territórios municipais do Mato Grosso do Sul à incidência da COVID-19. Verificou-se que dos 79 municípios, 73,41\% apresentam Alta Vulnerabilidade, e $74,7 \%$ foram classificados como Territórios de Risco Moderado a Alto Risco para a COVID-19, necessitando, portanto, de atenção ao planejamento territorial no enfrentamento da pandemia.
\end{abstract}

Palavras Chaves: Pandemia. Mapeamento. Ordenamento Territorial.

\begin{abstract}
In view of the current Pandemic situation declared by the World Health Organization in March 2020 , due to the rapid dispersion of Coronavirus, whose main disease - COVID-19 - may cause the overcrowding of public health services and accentuate the loss of life of population, questions arise regarding how vulnerable the territories are in relation to this dangerous phenomenon, and, in addition, what degree of risk the populations are exposed to, considering the characteristics of the territories. In this sense, the objective of this research was to analyze the Vulnerability and Risk to COVID-19 in the state of Mato Grosso do Sul, considering the characteristics of the territory, since that state presented an increase of $68 \%$ of confirmed cases in the epidemiological period between 23 and 30 April, reaching 255 contaminated. To achieve this objective, specific variables have been listed to quantify and to analyze both the Vulnerability and the risk of the municipal territories of Mato Grosso do Sul to COVID-19. It was found that of the 79 municipalities, $73,41 \%$ had High Vulnerability, and $74.7 \%$ were classified as Moderate to High Risk Territories for COVID-19, therefore needing attention to territorial planning in coping of Pandemic.
\end{abstract}

Key words: Pandemic. Mapping. Spatial Planning.

Recebido em: 02/05/2020

Aceito para publicação em: 21/05/2020 


\section{INTRODUÇÃO}

Desde o início de seu processo de estabelecimento enquanto sociedade organizada, o homem busca, das mais diversas formas, enquanto elemento biótico constituinte da paisagem, garantir a perpetuação da espécie tendo a dispersão e domínio dos territórios como sua principal estratégia biogeográfica. Figueiró (2015) aponta que os seres humanos alcançaram rapidamente uma distribuição cosmopolita no planeta, aliada principalmente a escassez de predadores no processo de competição, no entanto, frente a essa proliferação da espécie, na superfície terrestre, alguns fatores de origens biogeográficas ainda causam preocupações, sobretudo os que trazem como consequência a perda em massa dos indivíduos da espécie humana, tais como os riscos ligados a fatores biológicos.

De acordo com Organização Pan-americana de Saúde², a Organização Mundial da Saúde (OMS) declarou que o surto de doença causada pelo novo Coronavírus (COVID-19), constitui um grande risco para a humanidade, passando de Emergência de Saúde Pública de Importância Internacional - o mais alto nível de alerta da Organização, conforme previsto no Regulamento Sanitário Internacional, para a categoria de Pandemia, desde 11 de março de 2020.

Farias (2020), explica que o coronavírus é uma família de vírus que causa infecções respiratórias, conhecida desde a década de 1960. Um novo coronavírus foi descoberto em 31/12/19, após casos registrados em Wuhan, na China, e a doença foi chamada de COVID-19 ou Sars-Cov-22. A partir do território Chinês, a OMS divulga que foram confirmados no mundo, até 30 de abril de 2020, 3.090.445 casos de COVID-19 (71.839 novos em relação ao dia anterior) e 217.769 mortes (9.797 novas em relação ao dia anterior. Sendo que no Brasil - um dos países com transmissão comunitária da COVID19 - foram confirmados para essa mesma data, 85.380 casos e 5.901 mortes pela doença.

Penerliev e Petkov (2020) salientam que os espaços urbanos, sobretudo aqueles que configuram metrópoles, são em geral, os mais rapidamente afetados pelas consequências da dispersão do vírus, acrescentando ainda em suas conclusões a existência de uma correlação entre a população absoluta e a número de infectados. O Ministério da Saúde adverte que o principal vetor para a dispersão do vírus é o próprio Homem, sendo recomendado, como medidas de enfrentamento, política de diminuição de fluxos e aglomerações de pessoas nos territórios. Além de medidas de diminuição e/ou cancelamentos de fluxos aéreos e rodoviários, Cardoso et. al. (2020) acentua que o isolamento social vem sendo a medida mais usadas no enfrentamento da Pandemia, que indica a necessidade de compreender as características de cada território em relação aos fluxos e aglomeração de pessoa para entender o processo de disseminação do vírus e por consequência, da COVID-19.

Assim, no Brasil, são vistos como epicentro os Estados de São Paulo, Rio de Janeiro, Ceará, Amazonas e Distrito Federal. Ressalta-se que outros estados também sofrem com a contaminação de sua população pelo Coronavírus, dentre eles, o Mato Grosso do Sul que apresenta um avanço significativo dos casos confirmados, uma vez que, de acordo com informações da Secretaria Estadual de Saúde de Mato Grosso do Sul, no período epidemiológico de 23 a 30 de abril, apresentou um aumento de $68 \%$, atingindo 255 casos, com um índice de letalidade de 3,5\%.

Com base nessas informações surgem questionamentos relativos ao quão vulnerável os territórios estão em relação a esse fenômeno de perigosidade, e, para além disso, qual o Grau de Risco em que as populações estão expostas, considerando as características dos territórios.

De acordo com Braga et. al. (2006), o documento final da Conferência Mundial para a Redução de Desastres, em Kobe (Japão), em 2005, chama a atenção para a necessidade de se desenvolver sistemas de indicadores de risco e vulnerabilidade nos níveis nacional e subnacional como forma de permitir aos tomadores de decisão, um melhor diagnóstico das situações de risco e vulnerabilidade.

Nesse contexto, Souza e Lourenço (2015) afirmam que a Geografia vem tentando, ao longo do seu desenvolvimento epistemológico enquanto Ciência, aprimorar teorias e técnicas para a análise de riscos, em qualquer que seja a categoria: Natural, Ambiental ou Tecnológicos. Já Silva (2012) afirma que a compreensão e análise do risco, em qualquer que seja a dimensão e escala, desde que seja pelo viés da ciência geográfica, é importante pois, não só a consolidação do risco, mas também a percepção do mesmo causam alterações no espaço, possibilitando em caráter têmporo-espacial medidas de

\footnotetext{
${ }^{2}$ A Organização Pan-americana de saúde (OPAS) atua como escritório regional da Organização Mundial da Saúde (OMS) para as Américas e é a agência especializada em saúde do sistema interamericano

(https://www.paho.org/bra/)

DOl:http://dx.doi.org/10.14393/Hygeia0054402 Hygeia Edição Especial: Covid-19, Jun./2020 p.164 - 174, pág.165
} 
deteç̧ão, prevenção, mitigação e, quando da concretização do risco exige até mesmo a reorganização do território, materializam-se visivelmente na paisagem e no território.

Deste modo, a presente pesquisa tem por objetivo central, analisar a Vulnerabilidade e o Risco a COVID-19 no estado de Mato Grosso do Sul, considerando as características do território que se materializam como facilitadores de fluxo e aglomerações humanas, beneficiando a disseminação do coronavírus, bem como potencializando as consequências da dispersão da doença.

\section{METODOLOGIA}

$\mathrm{Na}$ presente pesquisa, a abordagem foca de forma contundente os conceitos de Risco e Vulnerabilidade sob a ótica da Ciência Geográfica. Nesse sentido, entende-se que para haver risco, tende haver o homem, uma vez que para essa ciência o Risco pode ser entendido como a probabilidade de um fenômeno perigoso trazer prejuízos (econômicos, à saúde, à vida, etc) ao homem, ou seja, essa concepção é comum a diversos autores tais como Marandola Jr. \& Hogan (2005), Almeida (2011), Silva (2011) e Souza \& Lourenço (2015).

Almeida (2011), por exemplo, afirma que é incontestável a onipresença do risco. Desde o momento em que a vida humana é concebida, correm-se diversos riscos. O risco é, assim, inerente à vida. Com a presença humana, o risco não deixa de existir (não existe "risco zero"), apenas varia no tempo e no espaço.

De acordo com Zêzere et al. (2006), o risco trata-se da probabilidade de ocorrência de um efeito específico causador de danos graves à humanidade e/ou ao ambiente, num determinado período e em circunstâncias determinadas. A autora ainda acrescenta que torna-se necessário compreender, portanto, que os fenômenos perigosos (naturais ou oriundos da organização social humana) geram a perigosidade a determinados elementos em risco (relacionados ao homem), no entanto, o grau de perigosidade está diretamente ligado à característica e/ou natureza do fenômeno, somado ainda a isto a vulnerabilidade do elemento em risco; sendo que, o grau de vulnerabilidade de cada elemento está diretamente relacionado às características do mesmo. Portanto, o risco pode ser quantificado e/ou avaliado pela relação entre a perigosidade e a vulnerabilidade.

Para Braga et. al. (2006), Vulnerabilidade é conceituada como uma combinação de variáveis que tornam uma população menos hábil para absorver o impacto de um evento perigoso e se recuperar dele ou poder contribuir para o recrudescimento da frequência, severidade, extensão ou imprevisibilidade do mesmo. Tais variáveis podem ser demográficas, econômicas, sociais, técnicas ou ambientais. Partindo do pressuposto comum de que é a vulnerabilidade que explica o porquê dos diferentes níveis de risco que diferentes grupos experimentam ao serem submetidos a perigos naturais de mesma intensidade.

Assim, a vulnerabilidade, numa definição alta, segundo Cutter (2011), é o potencial para a perda. A vulnerabilidade inclui, quer elementos de exposição ao risco (as circunstâncias que colocam as pessoas e as localidades em risco perante um determinado perigo), quer de propensão (as circunstâncias que aumentam ou reduzem a capacidade da população, da infraestrutura ou dos sistemas físicos para responder e recuperar de ameaças ambientais).

Braga et. al (2006) afirma que dada a complexidade das diferentes dimensões da vulnerabilidade, mensurá-las requer a integração de um grande número de informações relacionadas a uma pluralidade de disciplinas e áreas de conhecimento. Já Souza e Lourenço (2015) afirmam que ao efetuar análises e avaliações de risco dispomos de excelentes instrumentos de análise geográfica e espacial, vulgarmente designados por Sistemas de Informações Geográficas (SIG). Estas ferramentas possibilitam a entrada de múltiplas variáveis (camadas) quer do meio físico (relevo, vegetação, drenagem, precipitação), quer do meio social (população, ocupação do solo, pobreza, atividades econômicas, educação, etc.), de modo que estas fiquem sobrepostas facilitando a análise do fenômeno observado. Com base nessas premissas, essa pesquisa elencou distintas variáveis para a mensuração da escala do risco e vulnerabilidade do território sul-mato-grossense ao COVID-19, conforme Tabela 1:

A primeira etapa da proposta metodológica desta pesquisa foi identificação do grau de Vulnerabilidade de cada município do território sul-mato-grossense. Nesta etapa, considerando as informações de Souza \& Lourenço (2015), que indicam que a vulnerabilidade representa as condições determinadas por fatores ou processos físicos, sociais, econômicos e ambientais, que aumentam a susceptibilidade de uma comunidade ao impacto dos riscos. Portanto foram utilizados como critérios neste trabalho, variáveis que são consideradas características dos territórios que podem influenciar diretamente no aumento das consequências ligadas a perigosidade do fenômeno de risco, no caso, a Covid-19. 
Tabela 1 - Variáveis utilizadas na proposta metodológica deste trabalho como indicadores para a Vulnerabilidade e Risco da COVID - 19 em Mato Grosso do Sul.

\begin{tabular}{|c|c|c|c|}
\hline \multicolumn{2}{|c|}{ VULNERABILIDADE } & \multicolumn{2}{|c|}{ RISCO } \\
\hline Variável & Fonte de dados & Variável & Fonte de dados \\
\hline $\begin{array}{l}\text { Percentual de População } \\
\text { acima de } 60 \text { anos }\end{array}$ & $\begin{array}{l}\text { Instituto Brasileiro } \\
\text { de Geografia e } \\
\text { Estatística }\end{array}$ & $\begin{array}{l}\text { Distância das principais } \\
\text { Rodovias (BRs) }\end{array}$ & $\begin{array}{l}\text { Fonte primária } \\
\text { desta pesquisa }\end{array}$ \\
\hline $\begin{array}{l}\text { Leitos de UTI para cada } \\
10.000 \text { habitantes }\end{array}$ & $\begin{array}{l}\text { DataSUS/Ministério } \\
\text { da Saúde }\end{array}$ & $\begin{array}{l}\text { Distância das principais } \\
\text { fronteiras de Territórios } \\
\text { Contaminados }\end{array}$ & $\begin{array}{l}\text { Fonte primária } \\
\text { desta pesquisa }\end{array}$ \\
\hline $\begin{array}{c}\text { Número de Respiradores } \\
\text { para Cada } 10.000 \\
\text { habitantes }\end{array}$ & $\begin{array}{l}\text { DataSUS/Ministério } \\
\text { da Saúde }\end{array}$ & Demografia & $\begin{array}{l}\text { Instituto Brasileiro } \\
\text { de Geografia e } \\
\text { Estatística }\end{array}$ \\
\hline Comorbidades & $\begin{array}{l}\text { DataSUS/Ministério } \\
\text { da Saúde }\end{array}$ & & \\
\hline Taxa de Isolamento Social & $\begin{array}{l}\text { Secretaria Estadual } \\
\text { de Saúde de Mato } \\
\text { Grosso do Sul (em } \\
23 \text { de abril de } 2020 \text { ) }\end{array}$ & Grau de Vulnerabilidade & $\begin{array}{l}\text { Fonte primária } \\
\text { desta pesquisa }\end{array}$ \\
\hline
\end{tabular}

Assim, nesta pesquisa, como exposto na tabela 1 , foram elencadas como variáveis para a vulnerabilidade do território Sul-mato-grossense à Covid-19: a) projeção do percentual de população acima de 60 anos em 2019, tendo em vista que os números oficiais e estatísticos apresentados pela OMS apontam essa população como mais vulnerável à doença, sendo que, tal indicação é baseada nos casos asiáticos e europeus e mesmo com algumas ressalvas nas características da doença no Brasil, é verificado por meio dos Boletins sul-mato-grossense que para esse território também pode ser aplicada essa premissa; b) número de leitos de UTI e respiradores disponíveis na rede pública de saúde de cada município de Mato Grosso do Sul, para cada 10.000 habitantes, considerando-os, elementos infra estruturais de alto potencial de minimização das consequências mais graves e tratamentos da COVID-19 nos infectados; c) taxa de comorbidades, sendo aqui consideradas o percentual de população de diabéticos e hipertensos de cada município; e, d) taxa de isolamento, considerada pelos especialistas como estratégia mais adequada para diminuir a exposição da população à contaminação. Para essa variável, foi considerada a média de 15 dias de isolamento social ${ }^{3}$.

Apoiado nos trabalhos da Fundação Seade (1992a) e Waldvogel e Capassi (1998) para estimar o percentual da população acima de 60 anos em 2019, foram considerados os dados do Censo de 2000 e 2010, em que a partir destes identificou-se a TGCA (Taxa Geométrica de Crescimento Anual) para o intervalo entre os censos. Na sequência, foi aplicada a TGCA em uma função exponencial para calcular a projeção da população ao final do ano de 2019 , por fim realizamos uma soma apenas com a população acima de 60 anos.

Para a análise do Risco à Covid-19 no Mato Grosso do Sul, por sua vez, foram consideradas as recomendações de Braga et. al. (2006), de que a fórmula $R=V+P$ (risco = vulnerabilidade + perigo), e de que versões mais sofisticadas da mesma, tem sido usada em trabalhos que buscam mensurar riscos e vulnerabilidades a desastres naturais. Com base nessas premissas considerou-se nesta pesquisa primeiramente variáveis ligadas à iminência do contágio da população, considerando isso como relativo ao perigo da doença.

Assim, foram elencadas as variáveis: A) Distância das sedes dos municípios das principais rodovias de fluxos de transporte de cargas e pessoas no estado, sendo consideradas nessas circunstâncias, com base em Rech et. al (2017), as BRs (060, 163, 262, 267 e 419) as quais configuram eixos que ligam o território de análise aos Estados de São Paulo, Mato Grosso, Goiás e Paraná, além dos Países vizinhos Bolívia e Paraguai; B) Distância das principais fronteiras de territórios contaminados, sendo que no caso de Mato Grosso do Sul, a maior perigosidade está relacionada à proximidade com a fronteira com o Estado de São Paulo, por este ser um dos epicentros brasileiros da doença, e de acordo

${ }^{3}$ Foram utilizados dados de Taxa de Isolamento Social dos dias 22, 23, 26, 27, 28, 29 e 30 de Abril, e, 03, 04, 05 06, 07, 10, 12 e 13 de Maio de 2020, de acordo com disponibilização de dados pelo Governo do Estado de Mato Grosso do Sul, em https://www.coronavirus.ms.gov.br/ 
com dados de Radar Unesp COVID-194, a doença se alastra da capital para o interior (Sentido MS), pelas principais vias rodoviárias, as quais são ligadas diretamente à fronteira leste de Mato Grosso do Sul; C) Demografia, considerando apontamentos da OMS e do próprio Ministério da Saúde que indicam que o perigo da doença aumenta em cidades com maior quantitativo/concentração populacional, sendo que a escolha dessa variável ainda está de acordo com Penerliev e Petkov (2020) que afirmam um acorrelação entre a população absoluta e o número de casos confirmados, em estudo de disseminação espacial da COVID-19 ; e D) a vulnerabilidade, considerando as recomendações de Braga et. al. (2006) como já anunciado no início deste parágrafo.

Os dados levantados sobre as variáveis descritas foram organizados em colunas na plataforma Excel, sendo atribuído pesos de 1 a 3 de modo a representar o potencial de influência de cada variável na vulnerabilidade e/ou risco da COVID-19 no estado de Mato Grosso do Sul (Tabela 2). Com base na avaliação atribuída a cada variável, a pontuação adquirida foi usada em uma média aritmética simples ${ }^{5}$, para que os resultados também estivessem dentro da escala proposta de abordagem de 1 a 3.

Ainda na plataforma QGis, os dados quantitativos de casos confirmados da COVID-19 em Mato Grosso do Sul, extraídos do Boletim Epidemiológico - COVID-19, emitido pelo Governo de Estado de Mato Grosso do Sul (dados até 29/04 às 10h), foram também sobrepostos neste mapeamento, através de círculos proporcionais, de modo a avaliar a coerência dos resultados algébricos das variáveis elencadas para o Risco da COVID-19 com a realidade atual no território sul-mato-grossense

Tabela 2 - Escalas de influência de cada variável para a Vulnerabilidade e/ou o Risco do Covid-19 em cada município em Mato Grosso do Sul, sendo em geral 1 (baixo), 2 (Moderado) e 3 (alto).

\begin{tabular}{|c|c|c|c|}
\hline \multicolumn{2}{|c|}{ VULNERABILIDADE } & \multicolumn{2}{|c|}{ RISCO } \\
\hline Variável & Escala & Variável & Escala \\
\hline \multirow{3}{*}{$\begin{array}{l}\text { Leitos de UTI para cada } \\
10.000 \text { habitantes }\end{array}$} & 0 a $0,5=3$ & \multirow{3}{*}{$\begin{array}{l}\text { Distância das } \\
\text { Principais Rodovias }\end{array}$} & $\begin{array}{l}\text { Nas Margens da } \\
\text { Rodovia }=3\end{array}$ \\
\hline & 0,6 a $1,0=2$ & & $\begin{array}{l}\text { À até } 30 \mathrm{Km} \text { da } \\
\text { Rodovia =2 }\end{array}$ \\
\hline & Acima de 1,1 =1 & & $\begin{array}{c}\text { Acima de } 30 \mathrm{~km} \text { da } \\
\text { Rodovia }=1\end{array}$ \\
\hline \multirow{3}{*}{$\begin{array}{c}\text { Número de Respiradores } \\
\text { por } 10.000 \text { habitantes }\end{array}$} & 0 a $1,0=3$ & \multirow{3}{*}{$\begin{array}{c}\text { Distância da fronteira } \\
\text { do Estado de São } \\
\text { Paulo }\end{array}$} & Até $100 \mathrm{~km}=3$ \\
\hline & 1,1 a $2,9=2$ & & Entre 100 a $300 \mathrm{~km}=2$ \\
\hline & Acima de $3=1$ & & Acima de $300 \mathrm{~km}=1$ \\
\hline \multirow{3}{*}{$\begin{array}{c}\text { Taxa de isolamento social } \\
\text { em } 23 \text { de abril de } 2020\end{array}$} & $0 \%$ a $49 \%=3$ & \multirow{3}{*}{ Demografia } & Acima de 100.000 hab. \\
\hline & $50 \%$ a $69 \%=2$ & & 30.000 a 100.000 hab. \\
\hline & Acima de $70 \%=1$ & & Até 30.000 hab. \\
\hline \multirow{3}{*}{$\begin{array}{l}\text { Percentual de população } \\
\text { acima de } 60 \text { anos }\end{array}$} & Acima de $20 \%=3$ & \multirow{6}{*}{$\begin{array}{c}\text { Grau de } \\
\text { Vulnerabilidade }\end{array}$} & 2,34 a $3,00=3$ \\
\hline & $10,1 \%$ a $20 \%=2$ & & \\
\hline & 0 a $10 \%=1$ & & 1,67 a $2,33=2$ \\
\hline \multirow{3}{*}{ Comorbidades } & Acima de $20 \%=3$ & & \\
\hline & $10,1 \%$ a $20 \%=2$ & & 1 a $1,66=1$ \\
\hline & 0 a $10 \%=1$ & & \\
\hline
\end{tabular}

\section{RESULTADOS E DISCUSSÕES}

Os procedimentos adotados nesta pesquisa referentes à influência de cada uma das variáveis (população idosa, leitos de UTIs, respiradores, comorbidades e taxa de isolamento social) permitiram identificar a vulnerabilidade dos municípios do Mato Grosso do Sul frente à pandemia do Coronavírus e as possíveis consequências de sua proliferação e aumento dos casos da doença (COVID-19).

\footnotetext{
${ }^{4}$ Iniciativa de pesquisadores da Faculdade de Ciências e Tecnologia, Campus de Presidente Prudente da Universidade Estadual Paulista "Júlio de Mesquita Filho" (FCT-UNESP), referente à disseminação do novo coronavírus (COVID-19), em especial no interior paulista, cujos dados de mapeamentos da COVID-19 estão disponíveis em http://covid19.fct.unesp.br/.

${ }^{5}$ Além da Média aritmética também foram testadas média Geométrica além da Média ponderada, no entanto, diante do resultado final da aplicação, considerando a situação real de casos confirmados de contaminação no Território Sul-mato-grossense, até 15/05/2020, a Média Aritmética se mostrou mais adequada à realidade do território estudado.

DOl:http://dx.doi.org/10.14393/Hygeia0054402 Hygeia Edição Especial: Covid-19, Jun./2020 p.164 - 174, pág.168
} 
Deste modo, verificou-se que $73,41 \%$ dos municípios estão classificados como Muito Vulneráveis (Gráfico 1), sendo que os dois municípios mais importantes do estado, Campo Grande, Dourados, apresentaram baixa vulnerabilidade, e Três Lagoas e Corumbá que também são importantes centros regionais apresentaram Vulnerabilidade Moderada, à Covid -19. Tal classificação é pela infraestrutura hospitalar disponível pelo Sistema Único de Saúde disponíveis nestes municípios. A quantidade de Respiradores e UTIs disponíveis nesses territórios municipais é, em alguns casos, acima dos índices considerados satisfatórios pela OMS. No caso de Dourados e Campo Grande, essa situação é bem representada quando se considera por exemplo, a existência de hospitais universitários compondo o sistema infraestrutural de saúde no município.

No entanto, o cenário real desses municípios de grande porte, classificados como de baixa vulnerabilidade, muda ao considerarmos o sistema de Macrorregiões de Saúde utilizado pelo ministério da saúde, para a organização do Sistema Único de Saúde no Brasil, onde os municípios de porte regional, são considerados centros de atendimento da população dos municípios vizinhos. Essa situação eleva a quantidade de habitantes dependentes dos elementos UTls e Respiradores, considerados nessa pesquisa como variáveis infraestruturas de influência na vulnerabilidade dos territórios ao COVID-19.

Por outro lado, a grande maioria dos municípios de alta vulnerabilidade são aqueles de porte pequeno, com população inferior à 50.000 habitantes, cuja população possui elevado percentual de pessoas acima de 60 anos de idade; consideradas cidades de moradia de aposentados, com altos índices de população de baixa renda, os quais, em sua maioria, são acometidos por ao menos uma das comorbidades consideradas nesta pesquisa como influenciadoras da vulnerabilidade dos territórios sulmato-grossenses à COVID-19.

\section{Gráfico 1 - Vulnerabilidade dos Municípios de Mato Grosso do Sul à COVID-19}

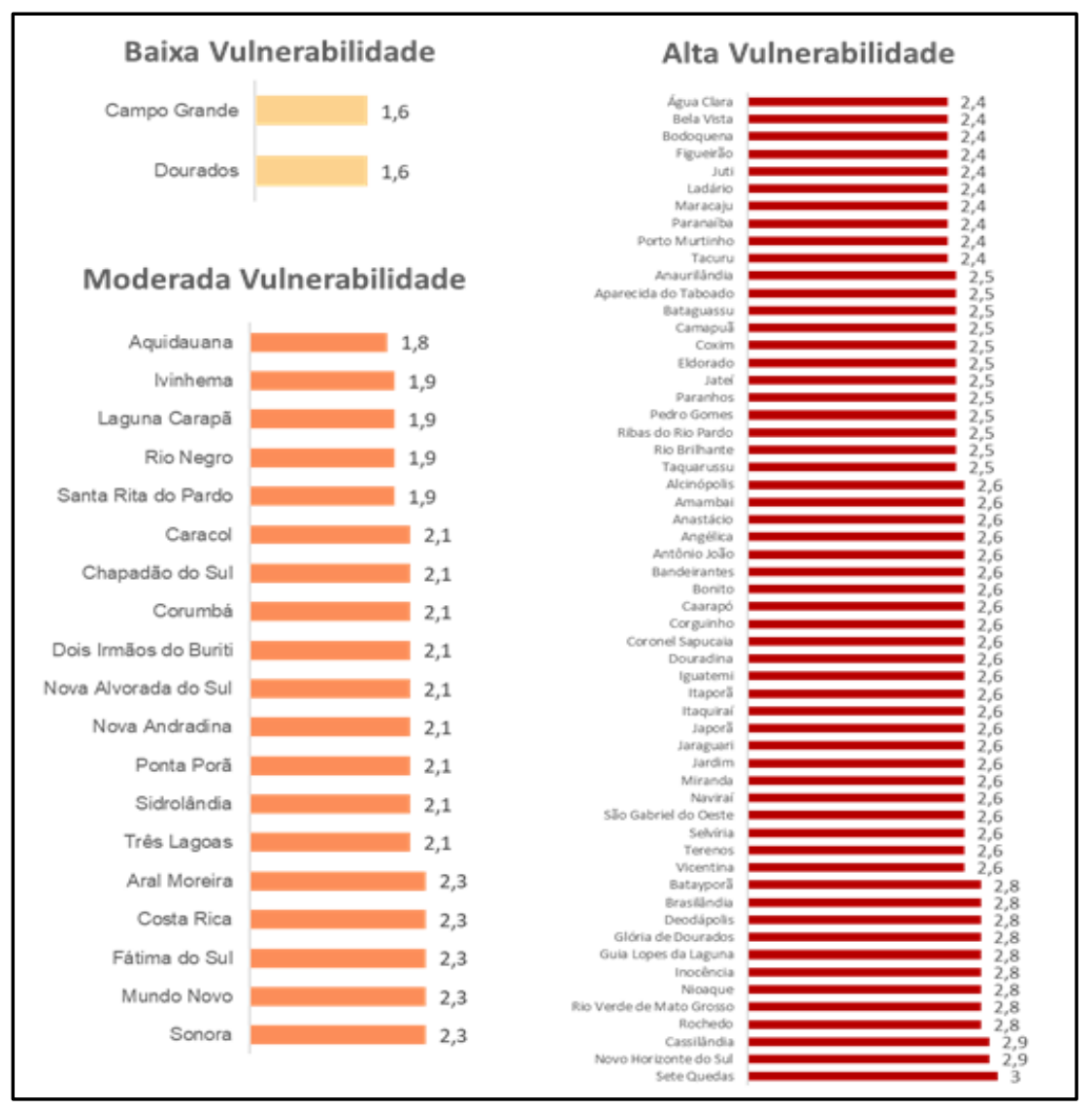

Faria (2020) acentua que a COVID-19 pode alcançar um número muito maior de infectados e de mortos ao se instalar nas áreas mais pobres do país: onde falta saneamento básico, principalmente água limpa para lavar as mãos; onde falta renda, e por isso as pessoas têm que se arriscar a descumprir o 
isolamento social para garantir algum sustento para a família; onde falta o Estado, e o sistema de saúde apresenta fragilidades. Oliveira e Morais (2020) complementam afirmando que diversos estudos publicados em revistas internacionais reportaram que as comorbidades mais prevalentes dos pacientes com COVID-19 são hipertensão e diabetes. Destaca-se que a grande maioria desses pacientes são idosos e fazem parte do grupo de risco da doença.

No entanto, alguns desses municípios são dependentes de cidades de grande porte, ao seu redor, consideradas de baixa vulnerabilidade, as quais podem contribuir para a amenização dos problemas relacionados à vulnerabilidade alta desses municípios, como é o caso dos municípios vizinhos a Campo Grande, Dourados e Três Lagoas (Figura 1).

Figura 1 - Espacialização da Vulnerabilidade dos Municípios de Mato Grosso do Sul à COVID-19 .

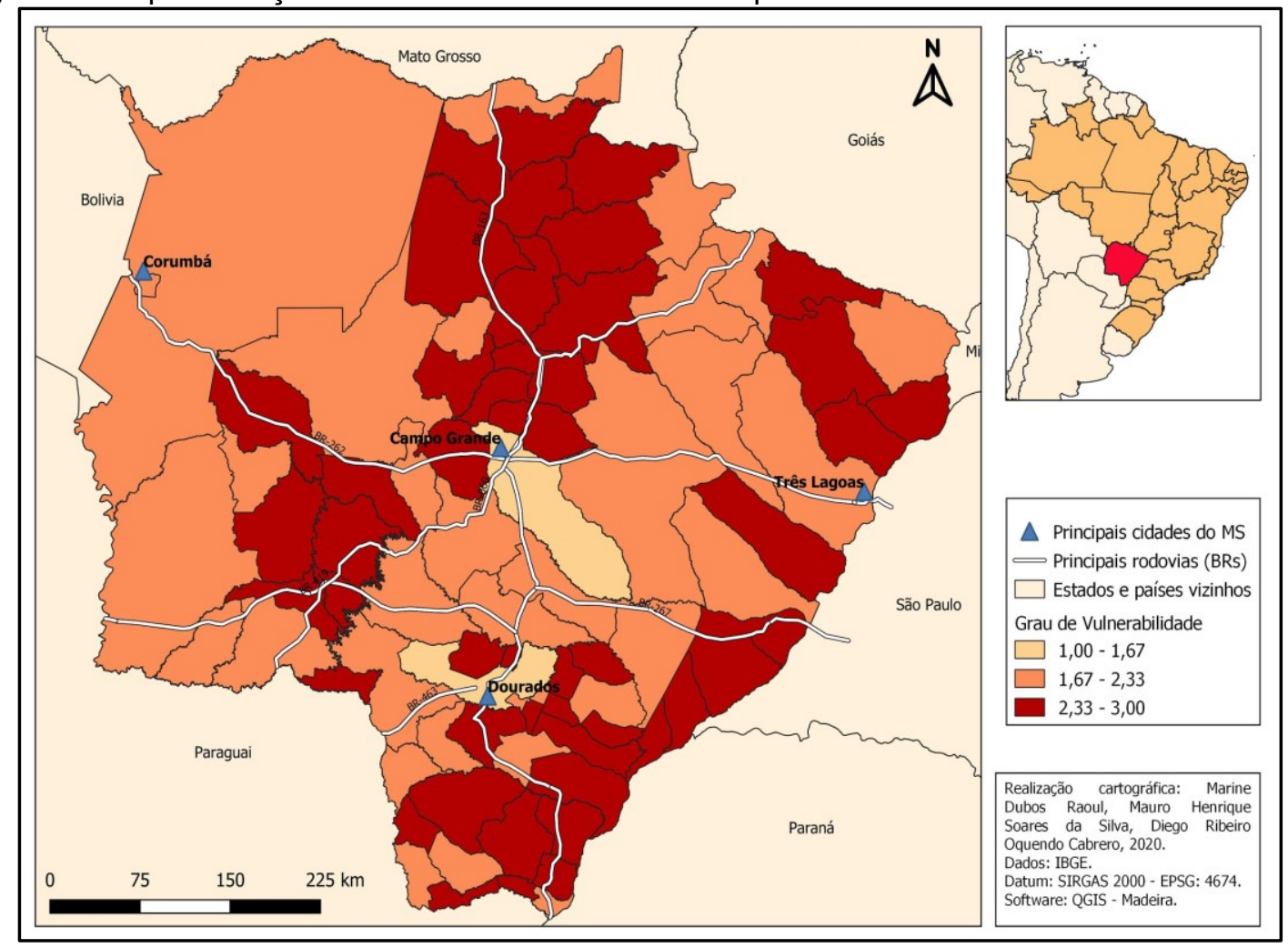

Ressalta-se ainda que dentre as variáveis adotadas nessa pesquisa, algumas delas são totalmente dependentes do planejamento territorial existente nessas localidades. Os investimentos na infraestrutura hospitalar, assim como as medidas de isolamento social levadas com mais precisão, podem alterar significativamente o grau de vulnerabilidade desses municípios.

Vale destacar que para Codeço (2020), a redução do fluxo intermunicipal só apresenta impacto significativo na disseminação para outras regiões se acompanhada de redução do contato entre indivíduos dentro de cada município, como medidas de distanciamento social. Se adotadas de maneira independente, ações locais se mostram mais relevantes do que a redução de fluxos intermunicipais.

Os dados obtidos nessa pesquisa permitem ainda afirmar que os municípios de grande porte no Mato Grosso do Sul, tais como Campo Grande, Dourados e Três Lagoas, que apresentam baixa vulnerabilidade à COVID-19, no caso dos dois primeiros, e vulnerabilidade moderada para o último, foram classificados como territórios de Alto Risco à doença, juntamente a outros dois municípios Bataguassu e Naviraí -, o que significa que as chances de acometimento do fenômeno perigoso nesses territórios são significativas (Gráfico 2). 
Gráfico 2 - Grau de Risco dos Municípios de Mato Grosso do Sul à COVID-19

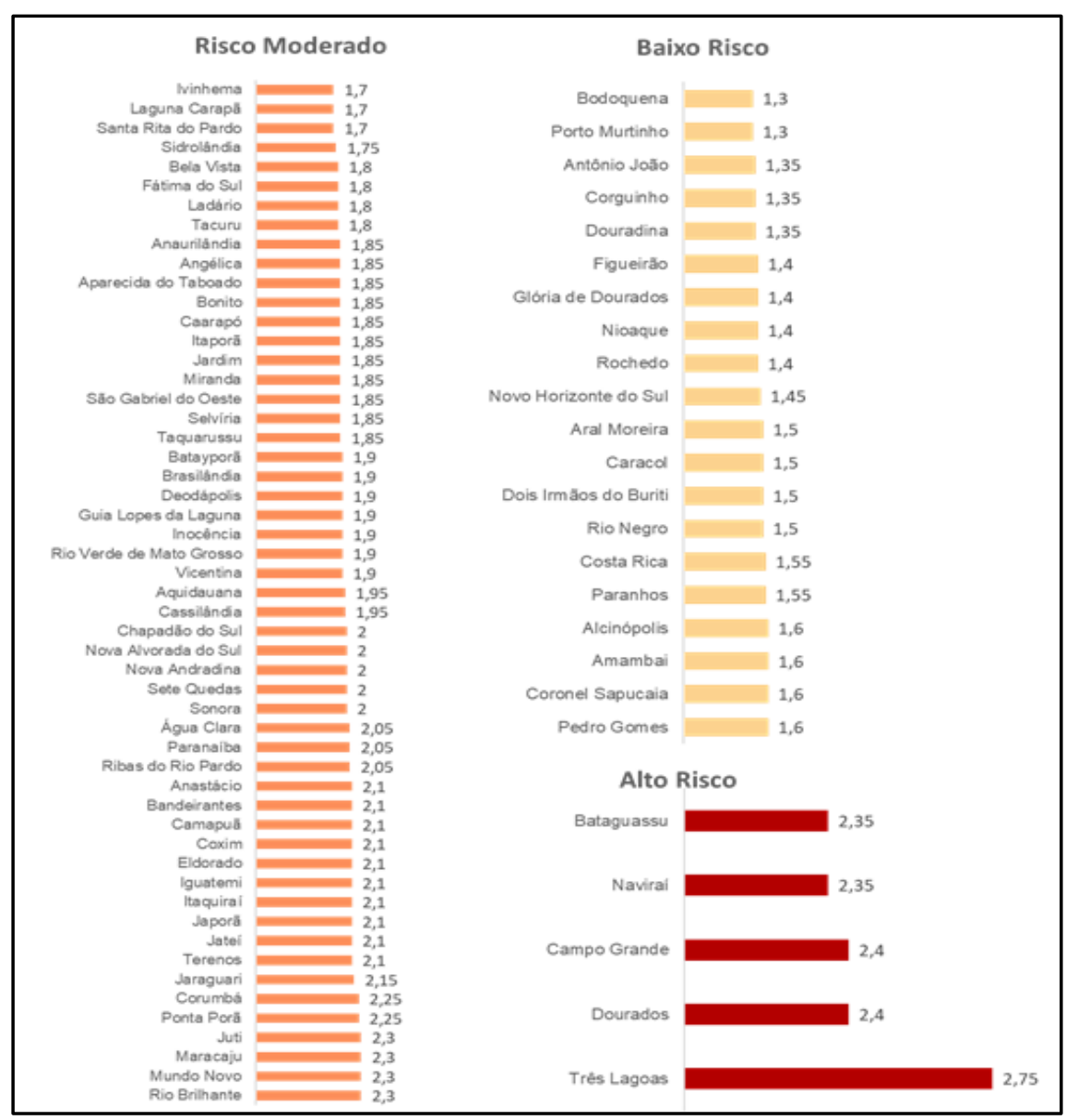

Ainda conforme o Gráfico 2 verifica-se que, dos 79 municípios de Mato Grosso do Sul 25,3\% apresentam Baixo Risco frente à COVID-19, enquanto os outros 74,7\% estão entre Risco Moderado e Alto Risco. Nesse contexto, dando destaque aos municípios de Três Lagoas, Campo Grande e Dourados, incluídos no grupo de municípios de Alto Risco, considerando a reflexão de que esses são centros de atendimento regional e portanto, responsáveis por vários outros municípios, em sua maioria classificados como de Risco Moderado, a situação nessas regiões pode se agravar ao passo que o planejamento territorial não crie medidas de amenização das vulnerabilidades territoriais à COVID-19.

Pires et.al (2020) aponta que a base da pirâmide social tem maior probabilidade de precisar de internação no caso de contaminação pela COVID-19, soma-se a isso outros fatores que tornam a população de baixa renda mais vulnerável a crise de saúde pública e ao colapso econômico associados à atual pandemia. Portanto, ainda segundo os autores, não basta dedicar esforços maiores para evitar a contaminação de idosos pela COVID-19: para evitar o colapso do sistema de saúde e a progressão acelerada do número de óbitos, as medidas desenhadas também devem destinar-se a proteger os mais pobres, seja por meio de políticas de preservação da renda que permitam o isolamento social, seja pela ampliação do número de leitos disponíveis no SUS.

Em alguns casos a situação pode ser ainda mais preocupante ao considerar que dentro de algumas Macrorregiões de Saúde, pode-se identificar mais de um município de Alto Risco constituindo o território. São os casos da Macrorregião de Três Lagoas, a qual está inclusa Bataguassu, e a Macrorregião de Dourados que comporta a população de Naviraí. Acrescenta-se ainda como exemplificação da necessidade de uma atenção ao planejamento territorial, o caso da Macrorregião de Saúde de Três lagoas a qual possui todos os seus municípios integrantes classificados como Risco Moderado ou Alto Risco.

Das variáveis elencadas para o estabelecimento do grau de risco dos territórios sul-mato-grossenses à COVID-19, duas delas se mostraram de extrema importância no que se refere à perigosidade relacionada aos aspectos espaciais da localização geográfica dos territórios, sendo elas a proximidade DOl:http://dx.doi.org/10.14393/Hygeia0054402 Hygeia Edição Especial: Covid-19, Jun./2020 p.164 - 174, pág.171 
das sedes do município com a fronteira leste do estado, divisa com o estado de São Paulo, além da distância geográfica destes com as principais rodovias de fluxo significativos de transporte de produtos e pessoas no estado.

Assim verificou-se que os municípios com Alto Risco à COVID-19 possuem relação com essas duas variáveis, sendo que Bataguassu, Campo Grande, Dourados, Naviraí e Três Lagoas, estão todos próximos à rodovias federais e localizados mais a Leste ou a Sudeste do Estado de Mato Grosso do Sul (Figura 2), apresentando direta relação com fluxos originados no Estado de São Paulo.

Figura 2 - Espacialização do Risco dos Municípios de Mato Grosso do Sul à COVID-19

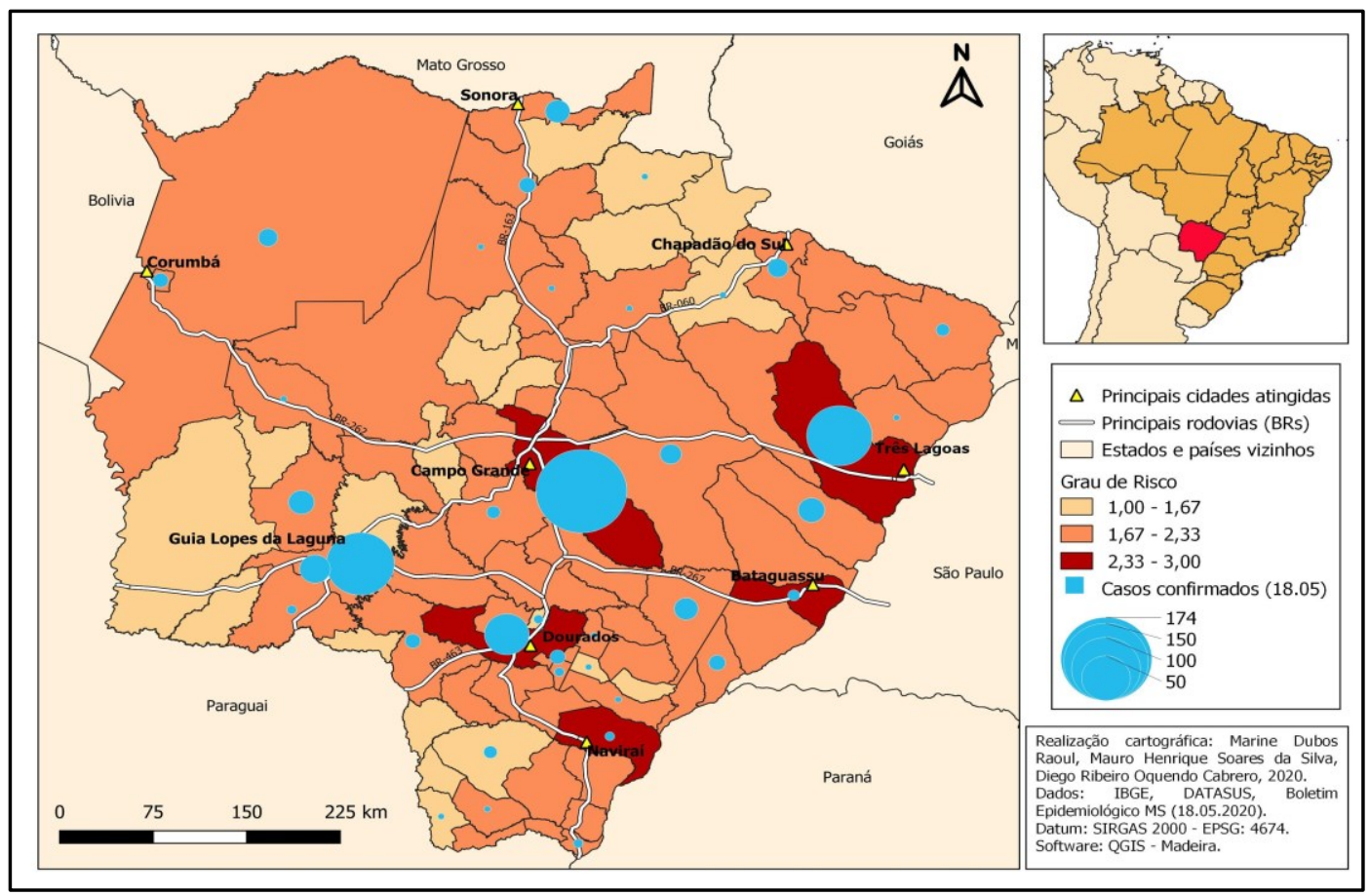

Por outro lado, a maior quantidade de municípios classificados com Baixo Risco à COVID-19 possui posição geográfica distante da fronteira leste do estado e das principais rodovias que atravessam o estado. Vale ainda citar o caso de Corumbá, o qual se materializa como um dos principais municípios do Mato Grosso do Sul, com um quantitativo populacional significativo, porém se apresentou com Risco Moderado à COVID-19, principalmente pelo seu distanciamento com a fronteira leste do Estado.

É importante ressaltar aqui, que a sobreposição dos casos de confirmados de COVID-19 em Mato Grosso do Sul com os resultados do grau de risco dos territórios municipais obtidos por meio dos procedimentos realizados nesta pesquisa, demonstram uma coerência com a realidade, uma vez que evidenciaram uma significativa relação com os territórios avaliados neste trabalho com Alto Risco ou Risco Moderado (Figura 2).

Dos municípios apontados nessa pesquisa como de Alto Risco, todos apresentaram casos confirmados. Naviraí apresenta dois casos e Bataguassu com 3 casos confirmados. Os demais municípios desta classe estão entre os mais contaminados do Estado, sendo Dourados com 67 casos, Campo Grande com 174 casos e Três Lagoas com 102 casos.

Ressalta-se ainda que dos quarenta municípios que apresentam casos de contaminação apenas $10 \%$ foram classificadas como de Baixo Risco à COVID-19. Nesse sentido, 90\% foram classificados nessa pesquisa como municípios de Alto Risco ou Risco Moderado, destacando Guia Lopes da Laguna com 105 casos, Jardim com 22, Bonito com 17, Sonora com 13 casos confirmados, Nova Andradina com 12 casos, confirmando que estas possuem características que acentuam a probabilidade de sofrer com o evento de perigosidade considerada nesta pesquisa - a COVID-19. 


\section{CONSIDERAÇÕES FINAIS}

A metodologia aplicada nesta pesquisa permitiu analisar a Vulnerabilidade e o Risco dos Territórios Municipais do Estado de Mato Grosso do Sul. As variáveis adotadas e a estratégia de aplicação de média aritmética simples permitiram a quantificação do grau de Vulnerabilidade, bem como do Risco, em uma escala de 1 a 3, classificável como Alto, Moderado ou Baixo para ambos indicadores.

Nesse sentido, os resultados permitiram avaliar que dos 79 municípios, $73,4 \%$ apresentam Alta Vulnerabilidade, e $74,7 \%$ foram classificados como Territórios de Risco Moderado a Alto Risco para a COVID-19. Sendo que dos municípios mais importantes do Estado, três deles merecem atenção especial, Três Lagoas, Dourados e Campo Grande, pois, juntos representam aproximadamente $44 \%$ da população do estado e ambos estão classificados como Alto Risco à COVID-19.

A espacialização dos dados também revelou que o fenômeno de perigosidade - a COVID-19 - no Mato Grosso do Sul é potencializado por fatores geográficos ligados à distância dos territórios em relação às rodovias de principais fluxos de cargas e pessoas, além da posição destes em relação à fronteira com o estado de São Paulo, a leste do Estado de Mato Grosso do Sul, o qual é um dos epicentros brasileiros desta pandemia e, favorecido pelas dinâmicas de suas vias rodoviárias, é uma significativa fonte de contaminação do território sul-mato-grossense.

Foi revelado também que o ordenamento do território pode ser uma estratégia de suma importância para a amenização das vulnerabilidades dos territórios e consequentemente, a mudança do grau de risco frente à COVID-19. Estas ações no ordenamento devem ter foco nas variáveis elencadas nesta pesquisa, que se configuram passíveis e dependentes das políticas públicas territoriais, nos diferentes níveis administrativos (Municipal, Estadual e/ou Federal), sendo essas variáveis: a taxa de isolamento social e disponibilidade de infraestrutura hospitalar, tais como leitos de UTIs e respiradores.

\section{BIBLIOGRAFIA}

ALMEIDA, Lutiana Queiroz. Por uma Ciência dos Riscos e Vulnerabilidades na Geografia. Revista Mercator, Fortaleza, v. 10, n. 23, p. 83-99, set./dez. 2011. https://doi.org/10.4215/RM2011.1023.0007

BRAGA, Tania Moreira; OIVEIRA, Elzira Lucia de; GIVISIEZ, Gustavo Henrique Naves. Avaliação de metodologias de mensuração de risco e vulnerabilidade social a desastres naturais associados à mudança climática. São Paulo em Perspectiva, v. 20, n. 1, p. 81-95, jan./mar. 2006. Disponível em: < https://wp.ufpel.edu.br/consagro/files/2011/03/BRAGA-Tania-Vulnerabilidade.pdf> Acessado em $23 / 04 / 2020$ às 20:30.

CARDOSO, Philipe Valente; SEABRA, Vinícius da Silva; BASTOS, Isabele Braz; COSTA, Evelyn de Castro Porto. A importância da análise espacial para tomada de decisão: um olhar sobre a pandemia de covid-19. Revista Tamoios, São Gonçalo (RJ), ano 16, n. 1, Especial COVID-19. pág. 125-137, maio 2020. https://doi.org/10.12957/tamoios.2020.50440

CODEÇO, Cláudia T; VILELA, Daniel; COELH, Flávio; BASTOS, Leonardo S; CARVALHO, Luiz Max; GOMES, Marcelo F; CRUZ, Oswaldo G; LANA, Raquel M. Estimativa de risco de espalhamento da COVID-19 nos estados brasileiros e avaliação da vulnerabilidade socioeconômica nos municípios. Relatório no 3 do Grupo de Métodos Analíticos de Vigilância Epidemiológica (MAVE), PROCC/Fiocruz e EMap/FGV -abril de 2020. Disponível em < https://covid-19.procc.fiocruz.br/> , Acessado em 25 de abril de 2020.

CUTTER, Susan L. A ciência da vulnerabilidade: modelos, métodos e indicadores. Revista Crítica de Ciências Sociais. N93. 2011. https://doi.org/10.4000/rccs. 165

FARIAS, Heitor Soares. $O$ avanço da Covid-19 e o isolamento social como estratégia para redução da vulnerabilidade. Revista Espaço e Economia (online), Volume 17.2020. https://doi.org/10.4000/espacoeconomia.11357

FIGUEIRÓ, Adriana. Biogeografia: dinâmicas e Transformações da natureza. São Paulo: Oficina de Texto, 2015.

FUNDAÇÃO SEADE. Projeções de população para as 11 regiões administrativas do Estado de São Paulo - 1970-1990 (Um Estudo Demográfico). Informe Demográfico. São Paulo, n.2, 1980.

MARANDOLA JR., Eduardo \& HOGAN, Daniel Joseph. Vulnerabilidades e riscos: entre geografia e demografia. Revista brasileira de Estudos Populacionais, São Paulo, v. 22, n. 1, p. 29-53, jan./jun. 2005 
OLIVEIRA, Orivan de Souza \& MORAIS, Arlandia Cristina Lima Nobre. COVID-19: uma pandemia que alerta à população. Interamerican Journal of Medicine and Health. Número 3, 2020. https://doi.org/10.31005/iajmh.v3i0.80

PENERLIEV, Milen e PETKOV, Veselin. Geodemographic Aspects of Covid-19. Revista Espaço e Economia (online), Volume 18. 2020. https://doi.org/10.4000/espacoeconomia.13444

PIRES, Luiza Nacif; CARVALHO, Laura; XAVIER, Laura Lima. COVID-19 e desigualdade: a Distribuição dos fatores de risco no Brasil. Experiment Findings. Abril/2020. Disponível em https://www.researchgate.net/publication/340452851. Acessado em 21 de abril de 2020.

RECH, Luiza Rodhen; MORAES, Manuela; CASAROTTO, Eduardo Luis; BINOTTO, Erlaine. Análise situacional das rodovias federais para o escoamento da produção do agronegócio em Mato Grosso do Sul e Mato Grosso. Revista do Centro de Ciências Econômicas e Informática (CCEI URCAMP), V.22, n37, 2017.

SILVA, Mauro Henrique Soares da Silva. Reflexões Sobre Riscos Ambientais: Diálogo entre as Geografias do Brasil e Portugal. Estudos do Quaternário, 8, APEQ, Braga (Portugal), 2012. https://doi.org/10.30893/eq.v0i8.65

SOUZA, Kátia Regina Góes \& LOURENÇO, Luciano. A Evolução do Conceito de Risco À Luz das Ciências Naturais e Sociais. Revista Territorium, n. ${ }^{\circ}$ 22, 2015. https://doi.org/10.14195/1647$\underline{7723221}$

ZÊZERE, J.L.; PEREIRA, A. RAMOS \& MORGADO, P. Perigos Naturais e Tecnológicos no Território de Portugal Continental. Revista Apontamentos de Geografia n. ${ }^{\circ}$ 19, CEG, Lisboa. 2006.

WALDVOGEL, B.; CAPASSI, R. Projeção populacional como instrumento de planejamento regional: o caso do Estado de São Paulo. In: ANAIS XI ENCONTRO NACIONAL DE ESTUDOS POPULACIONAIS. Belo Horizonte: Abep, 1998. 